\title{
A COUDÉ CAMERA FOR IMAGE-TUBE WORK
}

\author{
J. G. COHEN, J. E. SIMMONS, AND S. MANEY \\ Kitt Peak National Observatory, * Tucson \\ Received 1976 June 16
}

\begin{abstract}
A new camera designed specifically for image tubes and modern detectors has been installed at the coude focus of the 2.1-m telescope at Kitt Peak National Observatory. The optical and mechanical design as well as the performance of the camera are discussed.
\end{abstract}

Key words: instrumentation - coudé camera - image tubes

\section{Introduction}

A new coudé camera (camera no. 5) has been constructed at the 2.1-m telescope of Kitt Peak National Observatory (KPNO), which was designed specifically for use with image tubes and modern detectors, The previously available cameras (which are described in the KPNO User's Manual) were not suitable for image-tube work because of relatively inaccessible focal surfaces. Although camera no. 6 has been modified to permit the use of image tubes, it was not adequate for a number of reasons: work space was limited; it was difficult to operate; and since the focal length is long, the dispersion high, and the slit size small, using the coudé feed, the limiting magnitude, even with a two-stage tube, was 5 or 6 . Since the coude feed is now the primary source of light for the $2.1-\mathrm{m}$ coudé, image-tube use has become increasingly desirable. It was therefore felt that a completely new camera was necessary.

The design requirements were that the unvignetted field at the focal plane be $90 \mathrm{~mm}$ long and $20 \mathrm{~mm}$ high. The work area behind the focal plane and the back focal distance had to be as large as possible. It was decided that the focal ratio should be close to that of the present camera no. 3. The optical design was by J. E. Simmons and the mechanical design by S. Maney.

\section{Description of Camera}

The camera has a folded two-mirror configuration having less central obscuration than other systems considered previously. The main advantage is that the resulting unvignetted image surface is accessible behind the folding flat without length limitations. A horizontal bed with slides can accept most available image tubes and hardware.

Figure 1 shows the optical components, an aspheric corrector plate followed by a perforated tilted flat

\footnotetext{
* Operated by the Association of Universities for Research in Astronomy, Inc., under contract with the National Science Foundation.
}

sending the light back onto a concave spherical mirror. This mirror forms an image at $12 \mathrm{~cm}$ behind the rear surface of the tilted flat, which has a clearance bevel.t At the focal surface, a flattener lens corrects the field curvature found in this type design. Between the flat and the field flattener, there is enough room for magnets, shutters, and shields belonging to the image tubes. The optical design parameters are listed in Table I.

The aspheric corrector compensates the axial spherical aberration of the concave mirror and contributes enough coma and astigmatism of the right sign to obtain a good image across a 9 -cm field.

There are two corrector plates and field flatteners, namely a "red" and a "blue" set. The blue set is made of fused silica and optimized around $4200 \AA$ with good imaging from $3200 \AA$ to $5400 \AA$. The red pair is $\mathrm{BaK}$ 1 glass and optimized around $6500 \AA$ and capable of good performance from $5400 \AA$ to $8000 \AA$. In both sets a one-layer antireflection coating improves overall transmission and decreases parasitic light.

The camera has a focal length of $108 \mathrm{~cm}$ with a monochromatic $f / 3.6$ ratio and is capable of 5 degrees total field, or $90 \mathrm{~mm}$ at the focal surface. This field is adequate to cover the photocathode of most of the KPNO image tubes. For image tubes with a $40-\mathrm{mm}$ field and a resolution of about 40 lines $\mathrm{mm}^{-1}$, a field flattener is not necessary, as no degradation in the image quality is noted out to a $40-\mathrm{mm}$ field. A plateholder, which takes $3 \frac{1}{1}$-inch $\times 4$-inch plates, is also available for direct plate work and testing.

The mechanical layout behind the focal plane, which is illustrated in Figure 2, consists of a long work table on which two sets of rails or optical benches are mounted. The upper set of rails, which is removable, sits on the lower set. All of the present KPNO image tubes and the Kron tube can be mounted on one of

fUsers who wish to install their own detector, which requires a back focal distance of more than $9 \mathrm{~cm}$, should request a drawing of the clearance bevel and mirror mounting cell from the first author. 
these rail systems. There is a mechanical stop with a digital position encoder which can be moved along the rails. The first time the detector is used, it is set against the stop at focus. Thus if it is removed and then replaced, the stop is moved to the same encoder position and the detector again placed against the stop, so that the detector will again be close to focus. This capability for quick, repeatable setups in the "in focus" position has been tested and it performs well. There is extensive space for any detector that can meet the back focal distance criteria of less than 12

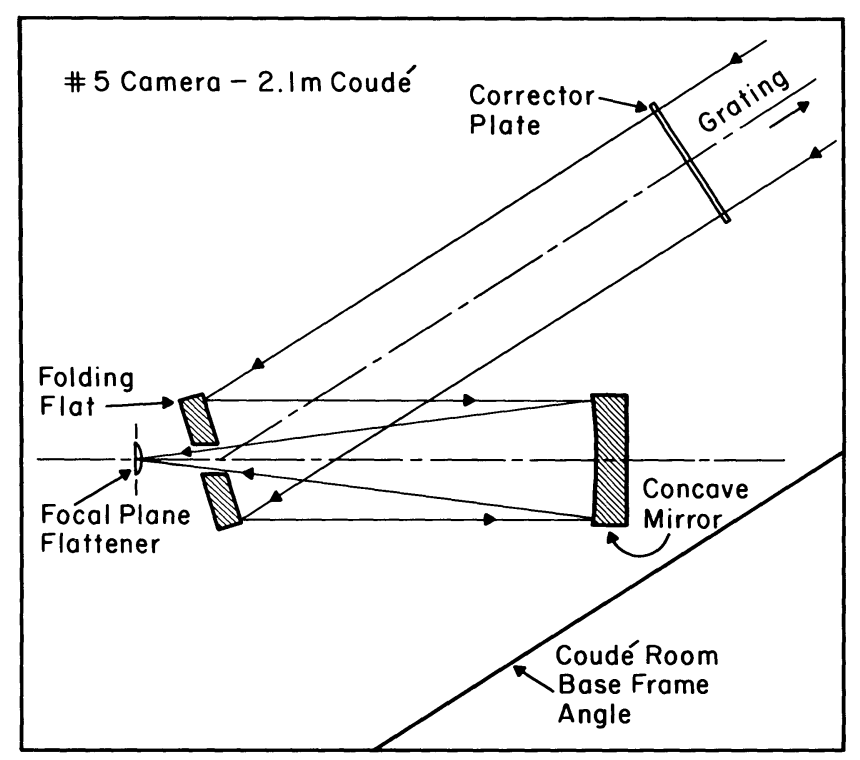

FIg. 1 -- Optical layout of no. 5 coudé image-tube camera. $\mathrm{cm}$, so that future use of Vidicons or other modern detectors is possible.

The detectors that have been used are (1) a twostage, cooled Carnegie image tube ( $\mathrm{S}-20$ cathode) which is permanently stored on a separate extension of the lower rails (see Fig. 2), and (2) a two-stage, cooled S-1 image tube. Both of these image tubes have a $40-\mathrm{mm}$ field. Although the Kron tube can be mounted on the rails and has only a $40-\mathrm{mm}$ field, it is not clear that it will function well without a field flattener.

\section{Performance of the No. 5 Camera}

Photographic test exposures made over the full 90$\mathrm{mm}$ field with both the red corrector plus red field flattener, and with the blue corrector plus blue field flattener, indicate outstanding imaging quality. There is no degradation in focus across the field once the tilt adjustment is set correctly. During photographic testing, it is possible to detect image quality changes with focal displacements of $0.05 \mathrm{~mm}$ of the concave spherical mirror. The observed full width at half maximum intensity of a $\mathrm{Ne}$ comparison line on an 098-04 emulsion (including the resolution of the emulsion, the intrinsic width of the line, and the spread function of the camera) is $14 \mu$. Since the resolution of the emulsion alone is most of that figure, the camera spread function is very small, and will be undetectable with any detector presently envisaged except possibly the Kron tube. In practice, the atmospheric absorption lines in stellar spectra and the emission comparison lines look equally sharp, so that there appears to be

TABIE I

\begin{tabular}{|c|c|c|c|c|c|}
\hline \multicolumn{6}{|c|}{ Design Parameters } \\
\hline Surface & $\begin{array}{l}\text { Type of } \\
\text { Surface }\end{array}$ & $\begin{array}{l}\text { Vertex } \\
\text { Radius } \\
\text { (cm) }\end{array}$ & $\begin{array}{c}\text { Disitance } \\
\text { to Next } \\
\text { Sul:face } \\
(\mathrm{cm})\end{array}$ & $\begin{array}{l}\text { Medium to } \\
\text { Next Surface }\end{array}$ & Remarks \\
\hline 1 & Grating & $\infty$ & 274.3 & Air & Aperture $30 \mathrm{~cm}$ \\
\hline 2 & Flat & $\infty$ & 1.0 & $\begin{array}{l}\text { Fused Silica } \\
\text { or } \\
\text { BaK-1 Glass }\end{array}$ & $\begin{array}{l}\text { Corrector plate } \\
\text { See NOTES }\end{array}$ \\
\hline $2 a$ & Aspherical & See NOTES & 131.4 & Air & See NOTES \\
\hline 3 & Flat & $\infty$ & $\varepsilon: 8.3$ & Air & Folding mirror \\
\hline 4 & Spherical & $216.0 \pm 1.0$ & 1017.4 & Air & \\
\hline 5 & Spherical & ${ }_{41.1^{34.5}} \pm 0.5$ & 0.6 & $\begin{array}{l}\text { Fused Silica } \\
\text { or } \\
\text { BaK-1 Glass }\end{array}$ & Field flattener \\
\hline $5 a$ & Flat & $\infty$ & 0.2 & Air & $\begin{array}{l}\text { For emulsion or } \\
\text { image tube best } \\
\text { focus must be } \\
\text { established }\end{array}$ \\
\hline 6 & Flat & $\infty$ & $\infty$ & -- & Focal Plane \\
\hline
\end{tabular}

NOTES: Surfaces $(2-2 a)$ and $(5-5 a)$ bound fused silica or BaK-1 glass for "blue" or "red" operation. If the meridian curve of surface $2 a$ is represented by $\mathrm{z}=\mathrm{a} \mathrm{y}^{2}+\mathrm{b} \mathrm{y}^{4}+\mathrm{c} \mathrm{y}^{6}$, then the coefficients are respectively $\mathrm{a}=-0.8863$ $\times 10^{-4}, \mathrm{~b}=+0.499 \times 10^{-7}, \mathrm{c}=+0.2337 \times 10^{-11}$ for the blue corrector; $\mathrm{a}=$ $-0.555 \times 10^{-4}, \mathrm{~b}=+0.429^{1} \times 10^{-7}, \mathrm{c}=+0.1028 \times 10^{-11}$ for the red corrector. 


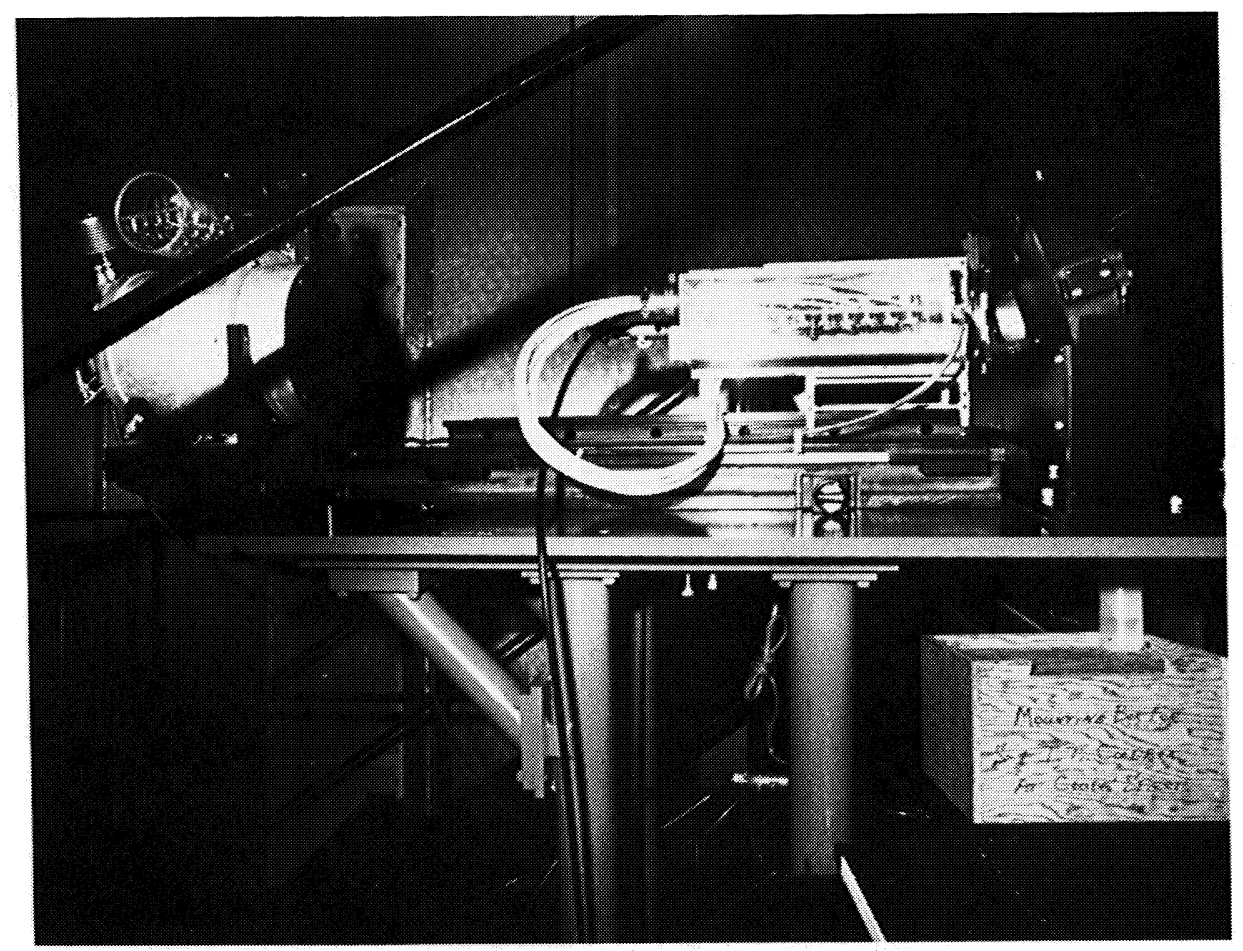

FIG. 2-View of work area behind focal plane of no. 5 image-tube camera. The Carnegie image-tube system is in its storage position; the $\mathrm{S}-1$ system is in position for use.

essentially no filling in of absorption lines by scattered light.

The speed gain of the no. 5 camera over the previously mentioned adaption of the no. 6 camera for image-tube use is significant. From a few exposures of selected objects with the S-1 and Carnegie image tubes, we estimate that a well-exposed spectrum 0.5 $\mathrm{mm}$ wide using a $25 \mu$ projected slit on a night with $2^{\prime \prime}-3^{\prime \prime}$ seeing can be obtained in 20 minutes at $5900 \AA$ using grating B (second order) (dispersion $20 \AA \mathrm{mm}^{-1}$ ) for $V=7.5$ using the Carnegie image tube. Under the same conditions, a spectrum $0.2 \mathrm{~mm}$ wide using the S-1 image tube at $9600 \AA$ using grating B (first order) for a star with $I=5$ requires approximately $30 \mathrm{~min}$ utes. With the 2.1-m telescope, $B=11.5$ is reached in 45 minutes at $4000 \AA$ with a dispersion of $13 \AA$ $\mathrm{mm}^{-1}$ on baked IIIa-J plates.

\section{Conclusion}

The new no. 5 image-tube camera at the 2.1 -m telescope of KPNO is functioning successfully, well within the design criteria. The limiting magnitude from test exposures is most encouraging. It is now available to visitors for use with the Carnegie system, and the S-1 system will also be available as soon as the remotely operated electronic shutter is completed.

\section{Note added in proof:}

The remotely operated electronic shutter for the S-1 image tube has been completed and installed. 